\title{
Simulation theory: An Introduction
}

\begin{abstract}
:
Navigating through social environment requires understanding and predicting complex interactions around us. This social understanding is in contrast to the understanding and predictions for inanimate objects that are governed by a set of fixed laws. Interactions with other individuals involves not only concrete observations like their height or physical appearance but their abstract states as emotions, beliefs, desires, intentions etc. Also most social interactions are dictated by judgments on such mental states than mere physical appearance. But how are humans able to achieve this? Considering that one individual doesn't have any physical access to other's mental states. And yet most individuals are highly adept at recognizing others' mental states. In the recent years, multiple insights in this direction have been provided by cognitive psychology and especially by cognitive neuroscience. Broadly categorizing, three main propositions have been suggested to explain how humans understand others - a theory-theory based account, a simulation and an interactionistic approach. The current work provides a brief account of the simulation theory.
\end{abstract}

1.1 Definition: Simulation theory (ST) in contrast to TT dismisses the idea that we use a specific 'theory' to understand other people's minds but rather we mentally simulate them. According to ST, to understand others, we utilize our own mental resources and ask the question "what would I have done if I were in his position" or, simply put we "put ourselves in their shoes". However, as argued by Gordon $(1986,1992)$, simulating other minds is not merely projecting our own situation but also accompanying it with 'necessary adjustments'. Thereby, the ST account is contrary to having a generalized mechanical law or a theory about the individuals around us. The ST has also been characterized as information poor mind reading (Goldman, 1992) as it does not require a huge set of information or theories as required by TT.

1.2 An example: Consider the case where you are playing a football match and see your opponent pass the ball in the opposite direction of the goal and you search for a possible explanation as to why he might have acted in this way. While searching you find that from the perspective of your opponent, there were none of his team members near the goal. Upon simulating what you would have done in such a case you arrive at the explanation that it would have been safer to pass the ball in the opposite direction which matched with the performed act. In this example, you use your own mental resources to comprehend other's intention based on the assumption that they would be similar to yours. It is also clear that this simulation required not just projecting oneself in another's situation but also making the necessary adjustments while taking into account the deviating goals and motivation of the other individual.

This explanation is contrary to the one that would be provided by a theory-theorists. A theory-theorists would explain this phenomenon by referring to the general assumption that 'when players don't find same team player next to them in the forward direction, they pass the ball to another same team player in the opposite direction'. 
1.3 Evidences for simulation theory: ST has been suggested to be routedin basic old and simple mechanisms, taking advantage, for instance, of human abilities to read gaze direction or to imitate others (Gallese \& Goldman, 1998; Gordon, 1992). Proponents of ST often cite examples of egocentric errors or biases - i.e influences of the mindreader's own mental states such as beliefs or desires in understanding others (Goldman \& Jordan, 2013) - to provide evidence for ST. ST has also found support from what is known as the "curse of knowledge" - a state where even though the participants are informed of the difference between their own knowledge and target's knowledge, participant's own knowledge seems to influence target's attributes (Birch \& Bloom, 2003; Camerer, Loewenstein, \& Weber, 1989; Nickerson, 1999).

ST has also gathered support from neurophysiological studies where, for instance, amygdala lesion in patients reduced their recognition of emotion in facial expressions (Adolphs, Tranel, Damasio, \& Damasio, 1994) as well as discovery of mirror neurons proposed to be a neural implementation of simulation (Gallese \& Goldman, 1998; Rizzolatti \& Craighero, 2004).

2.1 Formulation: ST has been difficult to assess because of the confusion in its conceptualization. There has been questions as to what could be termed as simulation. Different proponents of the theory have slightly different interpretations as to what would count as a simulation. Over the years, ST has been refined and reformulated. Goldman (2006) introduces simulation as a process in the following manner:

“Process $\mathrm{P}$ simulates process $\mathrm{P}^{\prime}$ iff (1) P duplicates, replicates or resembles $\mathrm{P}^{\prime}$ in some significant respect and (2) in its duplication of $\mathrm{P}^{\prime}, \mathrm{P}$ fulfills one of its purposes or functions. For mindreading simulation, $\mathrm{P}$ and $\mathrm{P}^{\prime}$ are mental processes and the purpose or function of $\mathrm{P}-$ the simulating process - is to understand a target's mental states".

2.2 Abstract or concrete: This definition obviously raises the questions as to what would constitute as "some significant respect". It has been argued that it is not sufficient for simulation to merely duplicate another process but it must involve a concretely similar processes (Fisher, 2006). A concrete replication would imply a similar set of systems or fine grained similarities in the replication. In contrast, an abstract replication might involve a different set of processes to replicate the given process.

Imagine for instance the case where, you would need to recognize a given human face. A human would utilize a specific set of processes while a computer uses a completely different set of rules to identify the given face. In this case, the computer is simulating the process in an abstract manner. (Fisher, 2006) and (Spaulding, 2010) claim that simulation as referred by Goldman and others has to simulate concretely to claim non-trivial effects.

Understanding this criteria is also important to clarify genuine cases of simulation compared to cases where a process just 'happens' to get the correct conclusion. Consider the case where your friend receives a gift certificate for a supermarket and you theorize that since he desperately needs a coffee machine and he is an Italian who really enjoys coffee, he would buy a coffee machine for himself. Suppose, in this case, your reasoning just so happens to be the same as your friend's (and he uses same reasons and buys a coffee machine). Despite the fact that you theorized, your reasoning process 
accidently resembled your friend's reasoning process. This similarity of processes has been referred to as an abstract similarity or abstract replication (Fisher, 2006). It is clear from the example that abstract replication would be entailed by at least some versions of TT.

2.3: Fulfilling function: It is also not clear when a process fulfills the function of another process? For a genuine simulation, one must distinguish between cases where a process happens to replicate another versus cases where the function of the process is to replicate. For example, in understanding where a tennis player will serve, I may act as a 'fact-finder' and theorize it based on the position where his opponent is standing. In this case, I use my visual system not for a simulation but for deriving a theory. So, even though, I successfully predict the outcome, my process merely happens to replicate the process going on in the target and thus would not be a simulation.

2.4: Strict formulation of simulation: In view of these points, it's imperative to develop a stricter formulation of what constitutes a simulation. The simulation, thus, needs to concretely replicate the target's process, i.e. have fine-grained similarities. The simulation also needs to be 'offline' utilizing non-standard inputs, i.e. it should utilize the same processes as in the target but without providing a final output in terms of an action. In addition, the inputs and outputs of the simulated procedure should also be the same as that of the target.

3.1 Classification : Having defined what a simulation needs to be, simulation could be classified on basis of:

1. Function - explanatory (retrodictive) or predictive (projective) or

2. Levels of mind reading - low or high.

3.2 Retrodictive and Projective simulations: Retrodictive simulation concerns the cases where explanations are generated after the behavior has been observed. Such simulations help us understand why an actor performed an action retrospectively (Gallese \& Goldman, 1998; Goldman, 1989; Spaulding, 2010).

Projective simulations, on the other hand are used to predict the consequences of others' actions. This takes in retrodictive simulation as an input. The aim here is to predict what an individual will do next (Gordon, 1992; Spaulding, 2010).

3.3 High level or low level simulation: A high level simulation concerns mental states of complex nature such as beliefs and propositional attitudes and has been defined to have at least some degree of accessibility to consciousness and voluntary control (Goldman, 2006). Earlier versions of ST were thought to be comprised only of such high level simulations. A high level simulation would consist of imagination in the usual sense where one consciously puts oneself in someone else's position and imagine what he/she would feel or do in such a scenario.

A low level simulation on the other hand, does not have conscious access or control over the simulation process. The simulation has been proposed to work unconsciously and automatically (Gordon, 1992). A neural realization of low level simulation has been proposed to be accomplished by an action observation/execution matching system ( mirror neuron system) (Gallese \& Goldman, 1998). 
3.3.1 Mirror neurons: This class of neurons, first discovered in monkeys, is active both while the individual performs an action and when the same individual observes another individual perform the same action (di Pellegrino, Fadiga, Fogassi, Gallese, \& Rizzolatti, 1992; Rizzolatti \& Craighero, 2004). It has been proposed that when we observe someone perform an action, activation in our mirror neuron system activates our brain 'as if' we were performing the same act. An inverse model that translates from observed actions to the respective motor commands is thought to play a role in determining the specific motor commands that may have caused that action (Kilner, Friston, \& Frith, 2007). Mirror neuron system, thus, has been proposed to be the basis for our understanding of others (Keysers \& Gazzola, 2009; Rizzolatti \& Craighero, 2004).

4.1 Limitations: Despite the supporting evidences for ST, there are many issues that face the theory:

4.1.1 Computational cost: This limitation primarily concerns high level simulation where one needs to track the possible mental states that caused the behavior. This can get relatively complex and computationally challenging given the complex social interactions involving multiple individuals and their interdependent behaviors.

4.1.2 Threat of collapse: Both low level and high level simulations for retrodictive case would loop infinitely and would have no stopping point as there is no way to confidently determine if we have arrived at the best explanation (Davies \& Stone, 1995)

4.2 Inapplicable cases: One can think of many cases where ST can't be applied. For instance, in cases of delusion of persecution or individuals who we know to exhibit idiosyncratic, irrational behavior, ST is irrelevant since our own experiences are too different from such individuals (Newen, 2015; Tversky \& Kahneman, 1974).

4.3 Argument from systematic errors: ST has also been criticized for not being able to explain the errors exhibited by children and adults (Saxe, 2005; Stich and Nichols 1995). For instance, egocentric errors in children for certain mental states are corrected later than others. It has been proven for instance that the mental ability to understand the perceptions and desires of other individuals occurs earlier in infantile development than the ability to ascribe belief which misrepresent the reality from the own point of view.(Gopnik \& Wellman, 1992).

4.4 Self vs other distinction: An "offline" simulation requires similar substrates as in the target would invariantly require the distinction that the action is performed by the other individual and not by the individual who is simulating. This distinction would require at least certain non-overlapping brain regions (Becchio, Adenzato, \& Bara, 2006).

5. Conclusions: Some of the criticisms of ST have led to the refinement of the theory. Strong versions of ST (pure simulation) have largely been dropped in favor of hybrid ST/TT models (Davies \& Stone, 1995; Goldman, 2006). Hybrid models with an input from theoretical knowledge from TT can solve the limitations of computational cost as well as threat of collapse. Hybrid models where simulation might not play an exhaustive role and proposing two or more cognitive processes driving simulation would explain different developmental time spans for beliefs vs desires (Goldman \& Sebanz, 2005; Goldman, 
2006). Despite these reformulations, ST and TT remain limited in the sense that they adopt a thirdperson perspective where the simulating individual is an observer (Gallagher, 2008). Our requirements for understanding others' actions, however, is critical while we are interacting with them rather than being in a passive retrospective condition. Both ST and TT accounts thus fail in explaining such situations.

\section{References:}

Adolphs, R., Tranel, D., Damasio, H., \& Damasio, A. (1994). Impared recognition of emotion in facial expressions following bilateral damage to the human amygdala. Nature. doi:10.1038/372669a0

Becchio, C., Adenzato, M., \& Bara, B. G. (2006). How the brain understands intention: different neural circuits identify the componential features of motor and prior intentions. Consciousness and Cognition, 15(1), 64-74. doi:10.1016/j.concog.2005.03.006

Birch, S. a J., \& Bloom, P. (2003). Children are Cursed: An Asymmetric Bias in Mental-State Attribution. Psychological Science, 14(3), 283-286. doi:10.1111/1467-9280.03436

Camerer, C., Loewenstein, G., \& Weber, M. (1989). The Curse of Knowledge in Economic Settings: An Experimental Analysis. Journal of Political Economy. doi:10.1086/261651

Davies, M., \& Stone, T. (1995). Mental simulation: Evaluations and applications. Readings in mind and language (Vol. 4).

di Pellegrino, G., Fadiga, L., Fogassi, L., Gallese, V., \& Rizzolatti, G. (1992). Understanding motor events: a neurophysiological study. Experimental Brain Research, 91(1), 176-80. Retrieved from http://link.springer.com/article/10.1007/BF00230027

Fisher, J. C. (2006). Does Simulation Theory Really Involve Simulation?*. Philosophical Psychology, 19(4), 417-432. doi:10.1080/09515080600726377

Gallagher, S. (2008). Direct perception in the intersubjective context. Consciousness and Cognition, 17(2), 535-43. doi:10.1016/j.concog.2008.03.003

Gallese, V., \& Goldman, A. (1998). Mirror neurons and the simulation theory of mind-reading. Trends in Cognitive Sciences, 2(12), 493-501. Retrieved from

http://www.sciencedirect.com/science/article/pii/S1364661398012625

Goldman, A. I. (1989). Interpretation Psychologized. Mind \& Language, 4, 161-185. doi:10.1111/j.1468-0017.1989.tb00249.x

Goldman, A. I. (1992). In Defense of the Simulation Theory. Mind \& Language, 7(1-2), 104-119. doi:10.1111/j.1468-0017.1992.tb00200.x

Goldman, A. I. (2006). Simulating Minds. Philosophical Books (Vol. 49). doi:10.1111/j.14680149.2008.459_15.x

Goldman, A. I., \& Jordan, L. C. (2013). Mindreading by simulation: The roles of imagination and 
mirroring. In Understanding Other Minds, 3rd ed. (pp. 448-466).

Goldman, A. I., \& Sebanz, N. (2005). Simulation, mirroring, and a different argument from error. Trends in Cognitive Sciences, 9(7), 320; author reply 321. doi:10.1016/j.tics.2005.05.008

Gopnik, A., \& Wellman, H. M. (1992). Why the child's theory of mind really is a theory. Mind and Language, 7.

Gordon, R. M. (1986). Folk Psychology as Simulation. Mind \& Language, 1(2), 158-171. doi:10.1111/j.1468-0017.1986.tb00324.x

Gordon, R. M. (1992). The Simulation Theory: Objections and Misconceptions. Mind \& Language, 7(1-2), 11-34. doi:10.1111/j.1468-0017.1992.tb00195.x

Keysers, C., \& Gazzola, V. (2009). Unifying Social Cognition. doi:10.1007/978-1-59745-479-7

Kilner, J. M., Friston, K. J., \& Frith, C. D. (2007). The mirror-neuron system: a Bayesian perspective. Neuroreport, 18(6), 619-23. doi:10.1097/WNR.0b013e3281139ed0

Newen, A. (2015). Understanding Others, 26. doi:10.15502/9783958570320

Nickerson, R. S. (1999). How we know - and sometimes misjudge - What others know: Imputing one's own knowledge to others. Psychological Bulletin, 125(6), 737-759. doi:10.1037/00332909.125.6.737

Rizzolatti, G., \& Craighero, L. (2004). The mirror-neuron system. Annual Review of Neuroscience, 27, 169-92. doi:10.1146/annurev.neuro.27.070203.144230

Saxe, R. (2005). Against simulation: the argument from error. Trends in Cognitive Sciences, 9(4), 1749. doi:10.1016/j.tics.2005.01.012

Spaulding, S. (2010). Simulation theory. Wiley Interdisciplinary Reviews: Cognitive Science, 1(4), 527-538. doi:10.1002/wcs.33

Tversky, A., \& Kahneman, D. (1974). Judgment under Uncertainty: Heuristics and Biases. Science (New York, N.Y.), 185(4157 (Sept. 27, 1974)), 1124-31. doi:10.1126/science.185.4157.1124 